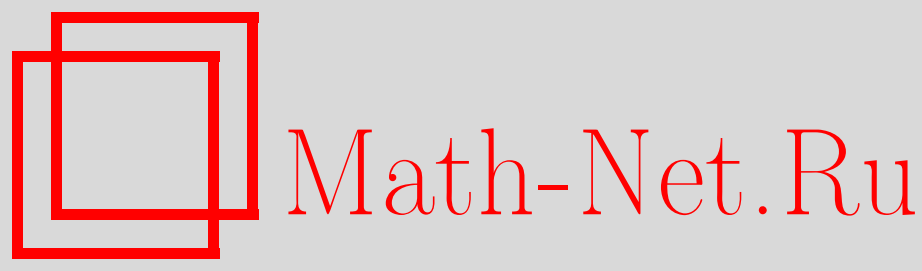

А. В. Латышев, А. А. Юшканов, Поведение плазмы с частотой столкновений, пропорциональной скорости электронов, во внешнем электрическом поле, ТМФ, 2007, том 153, номер 3, 409-421

DOI: https://doi.org/10.4213/tmf6145

Использование Общероссийского математического портала Math-Net.Ru подразумевает, что вы прочитали и согласны с пользовательским соглашением http: //www . mathnet.ru/rus/agreement

Параметры загрузки:

IP : 52.23 .180 .231

26 апреля 2023 г., 13:32:40

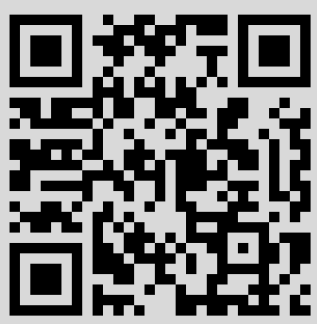




\title{
ФИЗИКА
}

Том 153, № 3

декабрь, 2007

2007 г.

А. В. Латышев*, А. А. Юшканов*

\section{ПОВЕДЕНИЕ ПЛАЗМЫ С ЧАСТОТОЙ СТОЛКНОВЕНИЙ, ПРОПОРЦИОНАЛЬНОЙ СКОРОСТИ ЭЛЕКТРОНОВ, ВО ВНЕШНЕМ ЭЛЕКТРИЧЕСКОМ ПОЛЕ}

\begin{abstract}
Аналитически решена задача о поведении газовой плазмы в полупространстве с использованием кинетического уравнения с частотой столкновений, пропорциональной модулю скорости электронов. Плазма находится во внешнем переменном электрическом поле. В качестве граничного условия используется зеркальное отражение электронов от границы плазмы. Решение используется для нахождения экранированного электрического поля.
\end{abstract}

Ключевые слова: столкновительная плазма, полупространство, зеркальное отражение, уравнения Власова-Максвелла.

\section{1. ПОСТАНОВКА ЗАДАЧИ}

Задача о поведении бесстолкновительной газовой плазмы (см. [1]) в полупространстве, находящемся во внешнем продольном (перпендикулярно поверхности) электрическом поле, для случая чисто зеркального отражения электронов от границы рассматривалась Ландау [2]. Более общий случай граничных условий рассмотрен в работе [3], где проводился анализ электрического поля на большом расстоянии от границы плазмы.

Аналитическое решение этой задачи представлено в наших работах [4]-[7]. В [4], [5] рассматривалась максвелловская плазма, а в [6], [7] - вырожденная, причем в [4], [6] рассматривались зеркальные граничные условия, а в [5], [7] - диффузные. В работе [2] рассматривался бесстолкновительный случай, а в работах [4]-[7] частота столкновений электронов считалась постоянной.

В работе [8] для описания поведения плазмы использовалось кинетическое уравнение с частотой столкновений, пропорциональной модулю скорости электронов, и было получено аналитическое решение задачи о скин-эффекте. В настоящей работе

* Московский государственный областной университет, Москва, Россия.

E-mail: avlatyshev@mail.ru,yushkanov@inbox.ru 
это уравнение используется для изучения поведения плазмы в полупространстве, находящемся во внешнем переменном электрическом поле $\mathbf{E}_{1}$. Откликом ионов на внешнее электрическое поле мы пренебрегаем [9].

Для описания функции распределения электронов $f(\mathbf{r}, \mathbf{v}, t)$ будем использовать кинетическое уравнение Власова [10] с самосогласованным электрическим полем и с интегралом столкновений в форме Бхатнагара-Гросса-Крука (БГК) с эффективной частотой столкновений электронов с частицами плазмы, пропорциональной модулю скорости электронов [8]:

$$
\frac{\partial f}{\partial t}+\mathbf{v} \frac{\partial f}{\partial \mathbf{r}}+e_{0} \mathbf{E} \frac{\partial f}{\partial \mathbf{p}}=\frac{v}{l}\left(f_{*}-f\right),
$$

где $e_{0}-$ заряд электрона, $f_{*}-$ максвелловская функция распределения,

$$
f_{*}=f_{*}(v)=n_{*}(\mathbf{r}, t)\left(\frac{\beta}{\pi}\right)^{3 / 2} e^{-\beta v^{2}}, \quad \beta=\frac{m}{2 k_{\mathrm{B}} T},
$$

$l$ - длина свободного пробега электронов, $k_{\mathrm{B}}$ - постоянная Больцмана, $m$ - масса электрона, $n_{*}$ - параметр, определяемый из закона сохранения числа частиц, $\mathbf{v}-$ скорость электрона, $\mathbf{p}$ - его импульс, $T$ - температура плазмы, которая считается постоянной, $\mathbf{E}=\mathbf{E}(\mathbf{r}, t)$ - самосогласованное электрическое поле внутри плазмы. Напряженность электрического поля $\mathbf{E}$ внутри плазмы удовлетворяет уравнению Максвелла

$$
\operatorname{div} \mathbf{E}=4 \pi e_{0} \int\left(f-f_{0}\right) d^{3} v,
$$

где $f_{0}=f_{0}(v)=n_{0}(\beta / \pi)^{3 / 2} e^{-\beta v^{2}}$ - абсолютный максвеллиан, соответствующий равновесному распределению электронов в отсутствие внешнего электрического поля.

Пусть максвелловская плазма занимает полупространство $x>0$, ось $x$ направлена перпендикулярно границе вглубь плазмы. Плазма находится во внешнем переменном электрическом поле $\mathbf{E}_{1}=\left(E_{0} e^{-i \omega t}, 0,0\right)$. Ввиду того что внешнее электрическое поле имеет одну ненулевую $x$-компоненту, зависимость функции распределения электронов от радиус-вектора $\mathbf{r}$ сводится к зависимости от координаты $x$ : $f=f(x, \mathbf{v}, t)$. По этой же причине электрическое поле внутри плазмы имеет вид $\mathbf{E}(x, t)=\left(E(x) e^{-i \omega t}, 0,0\right)$.

Теперь зададим граничные условия для уравнений (1.1) и (1.2). Рассеяние электронов на границе плазмы будем предполагать чисто зеркальным, т.е.

$$
f\left(0, v_{x}, v_{y}, v_{z}, t\right)=f\left(0,-v_{x}, v_{y}, v_{z}, t\right), \quad v_{x}>0 .
$$

Кроме того, на границе плазмы величина самосогласованного электрического поля совпадает с величиной внешнего поля, т.е.

$$
E(0)=E_{0} \text {. }
$$

Предположим, что напряженность электрического поля такова, что применимо линейное приближение [11]. Тогда функция распределения может быть представлена в виде

$$
f=f_{0}(v)+f_{1}(x, \mathbf{v}, t)
$$


где $f_{1}(x, \mathbf{v}, t)$ - линейное отклонение относительно абсолютного максвеллиана. В линейном приближении

$$
\begin{array}{r}
f_{*}=f_{0}+\frac{\delta n_{*}}{n_{0}} f_{0}, \quad \delta n_{*}=n_{*}-n_{0}, \\
\mathbf{E}(x, t) \frac{\partial f}{\partial \mathbf{p}}=\mathbf{E}(x, t) \frac{\partial f_{0}}{\partial \mathbf{p}}=-f_{0} \frac{v_{x} E(x) e^{-i \omega t}}{k_{\mathrm{B}} T} .
\end{array}
$$

С помощью (1.5) уравнения (1.1) и (1.2) принимают вид

$$
\begin{aligned}
\frac{\partial f_{1}}{\partial t}+v_{x} \frac{\partial f_{1}}{\partial x}-\frac{e_{0} v_{x}}{k_{\mathrm{B}} T} E(x) f_{0}(v) e^{-i \omega t} & =\frac{v}{l}\left(\frac{\delta n_{*}}{n_{0}} f_{0}(v)-f_{1}(x, \mathbf{v}, t)\right), \\
\frac{d E(x)}{d x} e^{-i \omega t} & =4 \pi e_{0} \int f_{1}(x, \mathbf{v}, t) d^{3} v
\end{aligned}
$$

Величину $\delta n_{*} / n_{0}$ найдем из закона сохранения числа частиц,

$$
\int v\left(f_{*}-f\right) d^{3} v \equiv \int v\left(\frac{\delta n_{*}}{n_{0}} f_{0}(v)-f_{1}(x, \mathbf{v}, t)\right) d^{3} v=0
$$

откуда

$$
\frac{\delta n_{*}}{n_{0}} \int v f_{0}(v) d^{3} v=\int v f_{1}(x, \mathbf{v}, t) d^{3} v .
$$

Замечая, что

$$
\int v f_{0}(v) d^{3} v=\frac{2 \pi}{\sqrt{\beta}}
$$

вместо (1.6) получаем следующее кинетическое уравнение:

$$
\begin{aligned}
\frac{\partial f_{1}}{\partial t} & +v_{x} \frac{\partial f_{1}}{\partial x}+\frac{v}{l} f_{1}(x, \mathbf{v}, t)= \\
& =\frac{e_{0} v_{x}}{k_{\mathrm{B}} T} E(x) f_{0}(v) e^{-i \omega t}+\frac{\sqrt{\beta} v}{2 \pi l} f_{0}(v) \int v^{\prime} f_{1}\left(x, \mathbf{v}^{\prime}, t\right) d^{3} v^{\prime}
\end{aligned}
$$

Граничное условие (1.3) на функцию $f$ согласно (1.5) имеет тот же вид и для функции $f_{1}$ :

$$
f_{1}\left(0, v_{x}, v_{y}, v_{z}, t\right)=f_{1}\left(0,-v_{x}, v_{y}, v_{z}, t\right), \quad v_{x}>0 .
$$

Из уравнений (1.7) и (1.8) видно, что функцию $f_{1}$ далее следует искать в виде

$$
f_{1}(x, \mathbf{v}, t)=f_{0}(v) h(x, \mathbf{v}) e^{-i \omega t} .
$$

С помощью (1.10) уравнения (1.7) и (1.8) упрощаются и приводятся к следующему виду:

$$
\begin{aligned}
v_{x} \frac{\partial h}{\partial x}+\left(\frac{v}{l}-i \omega\right) h(x, \mathbf{v}) & =\frac{e_{0} v_{x}}{k_{\mathrm{B}} T} E(x)+\frac{\sqrt{\beta} v}{2 \pi l} \int v^{\prime} f_{0}\left(v^{\prime}\right) h\left(x, \mathbf{v}^{\prime}\right) d^{3} v^{\prime} \\
\frac{d E(x)}{d x} & =4 \pi e_{0} \int f_{0}\left(v^{\prime}\right) h\left(x, \mathbf{v}^{\prime}\right) d^{3} v^{\prime}
\end{aligned}
$$


Умножим обе части (1.11) на $l \sqrt{\beta}$ и введем безразмерную координату $x_{1}=x / l$, скорость $\mathbf{C}=\sqrt{\beta} \mathbf{v}$ и безразмерное электрическое поле

$$
e\left(x_{1}\right)=\frac{e_{0} l}{k_{\mathrm{B}} T} E\left(x_{1}\right) .
$$

Кроме того, в интегралах в (1.11) и (1.12) перейдем к сферической системе координат и, считая, что функция $h$ зависит только от $x_{1}, \mu, C\left(\mu=C_{x} / C\right)$, проинтегрируем по азимуту - углу в плоскости $\left(C_{y}, C_{z}\right)$. С использованием безразмерных переменных и равенства (1.13) получаем следующую систему уравнений:

$$
\begin{aligned}
\mu C \frac{\partial h}{\partial x_{1}}+\left(C-i \omega_{1}\right) h\left(x_{1}, \mu, C\right)= & \frac{e_{0} l \mu C}{k_{\mathrm{B}} T} e\left(x_{1}\right)+ \\
& +C \int_{-1}^{1} \int_{0}^{\infty} e^{-C^{\prime 2}} C^{\prime 3} h\left(x_{1}, \mu^{\prime}, C^{\prime}\right) d \mu d C^{\prime}, \\
\frac{d e\left(x_{1}\right)}{d x_{1}}= & \frac{4 \omega_{1 \mathrm{p}}^{2}}{\sqrt{\pi}} \int_{-1}^{1} \int_{0}^{\infty} e^{-C^{\prime 2}} C^{\prime 2} h\left(x_{1}, \mu^{\prime}, C^{\prime}\right) d \mu^{\prime} d C^{\prime},
\end{aligned}
$$

где $\omega_{1 \mathrm{p}}=l \sqrt{\beta} \omega_{\mathrm{p}}, \omega_{1}=\omega l \sqrt{\beta}-$ безразмерная плазменная частота и частота внешнего электрического поля, соответственно, $\omega_{\mathrm{p}}$ - плазменная частота, $\omega_{\mathrm{p}}^{2}=4 \pi e_{0}^{2} n_{0} / \mathrm{m}$.

Далее безразмерную координату $x_{1}$ будем снова обозначать через $x$. Получаем следующую систему уравнений:

$$
\begin{aligned}
\mu \frac{\partial h}{\partial x}+\left(1-i \frac{\omega}{C}\right) h(x, \mu, C) & =\mu e(x)+\int_{-1}^{1} \int_{0}^{\infty} e^{-C^{\prime 2}} C^{\prime 3} h\left(x, \mu^{\prime}, C^{\prime}\right) d \mu^{\prime} d C^{\prime}, \\
\frac{d e(x)}{d x} & =\varkappa^{2} \int_{-1}^{1} \int_{0}^{\infty} e^{-C^{\prime 2}} C^{\prime 2} h\left(x, \mu^{\prime}, C^{\prime}\right) d \mu^{\prime} d C^{\prime},
\end{aligned}
$$

где $\varkappa^{2}=4 \omega_{1 \mathrm{p}}^{2} / \sqrt{\pi}$.

Зеркальное отражение электронов от границы (1.9) согласно (1.10) означает, что

$$
h(0, \mu, C)=h(0,-\mu, C), \quad \mu>0 .
$$

Условие на электрическое поле (1.4) согласно (1.13) имеет вид

$$
e(0)=e_{\mathrm{s}},
$$

где $e_{\mathrm{s}}=e_{0} l E_{0} /\left(k_{\mathrm{B}} T\right)$ - заданная величина.

Итак, исходная задача о поведении плазмы в полупространстве состоит в решении уравнений (1.15) и (1.16) с граничными условиями (1.17) и (1.18). Представим уравнение (1.16) в виде, который включает соответствующее граничное условие (1.18):

$$
\frac{d e(x)}{d x}=\varkappa^{2} \int_{-1}^{1} \int_{0}^{\infty} e^{-C^{\prime 2}} C^{\prime 2} h\left(x, \mu^{\prime}, C^{\prime}\right) d \mu^{\prime} d C^{\prime}+2 e(0) \delta(x),
$$

где $\delta(x)$ - дельта-функция Дирака. 


\section{2. АНАЛИТИЧЕСКОЕ РЕШЕНИЕ ЗАДАЧИ}

Решение системы (1.15), (1.19) ищем в виде интегралов Фурье:

$$
\begin{gathered}
h(x, \mu, C)=\frac{1}{2 \pi} \int_{-\infty}^{\infty} e^{i k x} \Phi(k, \mu, C) d k \\
e(x)=\frac{1}{2 \pi} \int_{-\infty}^{\infty} e^{i k x} E(k) d k, \quad \delta(x)=\frac{1}{2 \pi} \int_{-\infty}^{\infty} e^{i k x} d k .
\end{gathered}
$$

Подставим эти соотношения в уравнения (1.15) и (1.19) и получим систему характеристических уравнений:

$$
\begin{aligned}
\left(1-\frac{i \omega_{1}}{C}+i k \mu\right) \Phi(k, \mu, C) & =\mu E(k)+n_{1}(k), \\
i k E(k) & =\varkappa^{2} n_{0}(k)+2 e(0),
\end{aligned}
$$

где $n_{0}(k)$ и $n_{1}(k)$ - нулевой и первый моменты спектральной плотности $\Phi(k, \mu, C)$,

$$
n_{m}(k)=\int_{-1}^{1} \int_{0}^{\infty} e^{-C^{\prime 2}} C^{\prime m+2} \Phi\left(k, \mu^{\prime}, C^{\prime}\right) d \mu^{\prime} d C^{\prime}, \quad m=0,1 .
$$

Подставим $E(k)$ из $(2.4)$ в (2.3). Получаем уравнение, из которого находим

$$
\Phi(k, \mu, C)=-\frac{i \varkappa^{2} \mu n_{0}(k)+2 i \mu e(0)-k n_{1}(k)}{k\left(1-i \omega_{1} / C+i k \mu\right)} .
$$

Теперь воспользуемся определением моментов (2.5). Подставим (2.6) в (2.5) и получим систему дисперсионных уравнений:

$$
\begin{aligned}
& n_{0}(k)\left(k+i \varkappa^{2} T_{10}(k)\right)-n_{1}(k) k T_{00}(k)=-2 i e(0) T_{10}(k), \\
& n_{0}(k) i \varkappa^{2} T_{11}(k)+k n_{1}(k)\left(1-T_{01}(k)\right)=-2 i e(0) T_{11}(k),
\end{aligned}
$$

где

$$
T_{m l}(k)=\int_{-1}^{1} \int_{0}^{\infty} \frac{e^{-C^{2}} \mu^{m} C^{l+2}}{1-i \omega_{1} / C+i k \mu} d \mu^{\prime} d C^{\prime}, \quad m=0,1, \quad l=0,1 .
$$

Из системы дисперсионных уравнений получаем

$$
\begin{aligned}
& n_{0}(k)=-2 i e(0) \frac{T_{10}(k)\left(1-T_{10}(k)\right)+T_{00}(k) T_{11}(k)}{k\left(1-T_{01}(k)\right)+i \varkappa^{2}\left(T_{10}(k)+D(k)\right)}, \\
& n_{1}(k)=-2 i e(0) \frac{T_{11}(k)}{k\left(1-T_{01}(k)\right)+i \varkappa^{2}\left(T_{10}(k)+D(k)\right)},
\end{aligned}
$$

где

$$
D(k)=T_{00}(k) T_{11}(k)-T_{01}(k) T_{10}(k) .
$$

Найдем $E(k)$ и $\Phi(k, \mu, C)$. Имеем

$$
\begin{gathered}
E(k)=-\frac{2 i e(0)}{\Lambda(k)}, \\
\Phi(k, \mu, C)=\frac{E(k)}{1-i \omega_{1} / C+i k \mu}\left[\mu+\frac{T_{11}(k)}{1-T_{01}(k)}\right],
\end{gathered}
$$


где $\Lambda(k)$ - дисперсионная функция,

$$
\Lambda(k)=k+i \varkappa^{2}\left[T_{10}(k)+\frac{T_{00}(k) T_{11}(k)}{1-T_{01}(k)}\right] .
$$

Теперь решение системы (1.15), (1.19) найдем в виде интегралов Фурье, подставляя (2.7) в равенства (2.1) и (2.2):

$$
\begin{gathered}
h(x, \mu, C)=\frac{1}{2 \pi} \int_{-\infty}^{\infty} \frac{e^{i k x} E(k)}{1-i \omega_{1} / C+i k \mu}\left[\mu+\frac{T_{11}(k)}{1-T_{01}(k)}\right] d k, \\
\frac{e(x)}{e(0)}=\frac{1}{\pi i} \int_{-\infty}^{\infty} \frac{e^{i k x}}{\Lambda(k)} d k .
\end{gathered}
$$

В интегралах $T_{m n}(k)$, входящих в выражения $(2.8)$ и $(2.9)$, можно вычислить внутренние интегралы. Выделим действительную и мнимую части в первом интеграле:

$$
J_{0}(C, k)=\int_{-1}^{1} \frac{d \mu}{1-i \omega_{1} / C+i k \mu}=L_{1}(C, k)-i L_{2}(C, k),
$$

где

$$
\begin{aligned}
& L_{1}(C, k)=\frac{1}{k} \operatorname{arctg}\left(k-\frac{\omega_{1}}{C}\right)+\frac{1}{k} \operatorname{arctg}\left(k+\frac{\omega_{1}}{C}\right), \\
& L_{2}(C, k)=\frac{1}{2 k} \ln \frac{1+\left(k-\omega_{1} / C\right)^{2}}{1+\left(k+\omega_{1} / C\right)^{2}} .
\end{aligned}
$$

Для второго интеграла

$$
J_{1}(C, k)=\int_{-1}^{1} \frac{\mu d \mu}{1+i k \mu-i \omega_{1} / C}=M_{1}-i M_{2}
$$

также найдем действительную и мнимую части:

$$
\begin{aligned}
& M_{1}(C, k)=\frac{1}{k}\left(L_{2}(C, k)+\frac{\omega_{1}}{C} L_{1}(C, k)\right), \\
& M_{2}(C, k)=\frac{1}{k}\left(2-L_{1}(C, k)+\frac{\omega_{1}}{C} L_{2}(C, k)\right) .
\end{aligned}
$$

Теперь интегралы $T_{m n}(k)$ имеют следующий вид:

$$
\begin{array}{ll}
T_{0 j}(k)=\int_{0}^{\infty} e^{-C^{2}} C^{2+j}\left(L_{1}(C, k)-i L_{2}(C, k)\right) d C, & j=0,1, \\
T_{1 j}(k)=\int_{0}^{\infty} e^{-C^{2}} C^{2+j}\left(M_{1}(C, k)-i M_{2}(C, k)\right) d C, & j=0,1 .
\end{array}
$$

\section{3. МОДЫ ДРУДЕ И ДЕБАЯ}

В силу нечетности функции $\Lambda(k)$ ее разложение при малых $k(k \rightarrow 0)$ имеет вид $\Lambda(k)=\Lambda^{\prime}(0) k+\cdots$, где

$$
\Lambda^{\prime}(0)=1+\frac{2}{3} \varkappa^{2}\left[s_{0}\left(\omega_{1}\right)+\frac{2 t_{0}\left(\omega_{1}\right) s_{1}\left(\omega_{1}\right)}{1-2 t_{1}\left(\omega_{1}\right)}\right],
$$


причем в (3.1) входят интегралы

$$
\begin{array}{ll}
t_{k}\left(\omega_{1}\right)=\int_{0}^{\infty} \frac{e^{-C^{2}} C^{k+3}}{C-i \omega_{1}} d C, & k=0,1,2, \\
s_{k}\left(\omega_{1}\right)=\int_{0}^{\infty} \frac{e^{-C^{2}} C^{k+4}}{\left(C-i \omega_{1}\right)^{2}} d C, & k=0,1 .
\end{array}
$$

Замечая, что

$$
1-2 t_{1}\left(\omega_{1}\right)=-i \omega_{1} 2 t_{0}\left(\omega_{1}\right), \quad \omega_{1} s_{0}\left(\omega_{1}\right)+i s_{1}\left(\omega_{1}\right)=i t_{1}\left(\omega_{1}\right)
$$

преобразуем (3.1) к виду

$$
\Lambda^{\prime}(0)=1+i \frac{2 \varkappa^{2}}{3 \omega_{1}} t_{1}\left(\omega_{1}\right)
$$

Теперь распределение электрического поля в полупространстве (2.9) можно представить в виде

$$
\frac{e(x)}{e(0)}=\frac{1}{\pi i} \int_{-\infty}^{\infty} e^{i k x}\left[\frac{1}{\Lambda(k)}-\frac{1}{\Lambda^{\prime}(0) k}\right] d k+\frac{1}{\Lambda^{\prime}(0) \pi i} \int_{-\infty}^{\infty} \frac{e^{i k x}}{k} d k,
$$

или, вычисляя второй интеграл,

$$
\frac{e(x)}{e(0)}=\frac{1}{\Lambda^{\prime}(0)}+\frac{1}{\pi i} \int_{-\infty}^{\infty} e^{i k x}\left[\frac{1}{\Lambda(k)}-\frac{1}{\Lambda^{\prime}(0) k}\right] d k .
$$

Величина $1 / \Lambda^{\prime}(0)$ в (3.3) имеет глубокий физический смысл. Покажем, что величина $\Lambda^{\prime}(0)$ в точности совпадает с продольной диэлектрической проницаемостью плазмы в пределе $k \rightarrow 0$. В этом случае продольная диэлектрическая проницаемость плазмы $\varepsilon(\omega)$ может быть выражена через высокочастотную диэлектрическую проводимость плазмы, найденную в работе [8]:

$$
\varepsilon(\omega)=1+\frac{4 \pi i}{\omega} \sigma_{0} 2 t_{1}(0.75 \sqrt{\pi} \omega \tau),
$$

где $\sigma_{0}$ - статическая проводимость плазмы [9],

$$
\sigma_{0}=\frac{e_{0}^{2} n_{0} \tau}{m}
$$

$\tau$ - время свободного пробега электронов.

Отметим, что в используемое кинетическое уравнение входит длина свободного пробега электронов $l$. Связь между величинами $l$ и $\tau$ определяется тем требованием, чтобы выражения для статической проводимости, записанные через $l$ и через $\tau$, совпадали. Используемый интеграл столкновений приводит к следующему выражению для статической проводимости [8]:

$$
\sigma_{0}=\frac{2 e_{0}^{2} n_{0} k}{3 \sqrt{\pi \beta} k_{\mathrm{B}} T}
$$


Из сравнения (3.5) и (3.6) получаем связь между $l$ и $\tau$ :

$$
l=\frac{3}{4} \sqrt{\frac{\pi}{\beta}} \tau .
$$

Введем следующие обозначения соответственно для безразмерной частоты внешнего электрического поля и безразмерной частоты столкновений электронов:

$$
\gamma=\frac{\omega}{\omega_{\mathrm{p}}}, \quad \varepsilon=\frac{\nu}{\omega_{\mathrm{p}}}, \quad \nu=\frac{1}{\tau} .
$$

Вернемся к доказательству равенства выражений (3.2) и (3.4). Требуется показать, что

$$
\frac{8 \pi \sigma_{0}}{\omega}=\frac{2 \varkappa^{2}}{3 \omega_{1}} \text {. }
$$

Используя (3.7), получаем $\omega_{1}=\omega \sqrt{\beta} l=0.75 \sqrt{\pi} \omega / \nu$. На основании (3.7) и (3.8) получаем

$$
\frac{2 \varkappa^{2}}{3 \omega_{1}}=\frac{8 \omega_{1 \mathrm{p}}^{2}}{3 \sqrt{\pi} \omega_{1}}=\frac{8 \omega_{\mathrm{p}}^{2}(l \sqrt{\beta})}{3 \sqrt{\pi} \omega}=\frac{2 \omega_{\mathrm{p}}^{2}}{\omega \nu}=\frac{2}{\gamma \varepsilon} .
$$

С другой стороны, учитывая, что $\sigma_{0}=\omega_{\mathrm{p}}^{2} /(4 \pi \nu)$, имеем

$$
\frac{8 \pi \sigma_{0}}{\omega}=\frac{2 \omega_{\mathrm{p}}^{2}}{\omega \nu}=\frac{2}{\gamma \varepsilon}
$$

что и требовалось показать.

Из уравнения (1.15) найдем отвечающую электрическому полю $e_{0}(x)=e(0) / \Lambda^{\prime}(0)$ функцию распределения электронов:

$$
h_{0}(x, \mu, C)=\frac{e(0) \mu C}{\Lambda^{\prime}(0)\left(C-i \omega_{1}\right)} .
$$

Таким образом, выделенное частное решение системы Власова-Максвелла имеет следующий вид: $h=h_{0}(x, \mu, C), e=e_{0}(x)$. Это частное решение естественно назвать модой Друде (см. [9]). Она описывает объемную проводимость.

Функцию распределения также можно представить в виде, в котором выделена мода Друде:

$$
\begin{aligned}
\frac{h(x, \mu, C)}{e(0)}= & \frac{\mu C}{\left(C-i \omega_{1}\right) \Lambda^{\prime}(0)}+ \\
& +\frac{1}{\pi i} \int_{-\infty}^{\infty} \frac{e^{i k x}}{1+i\left(k \mu-\omega_{1} / C\right)}\left[\mu+\frac{T_{11}(k)}{1-T_{01}(k)}\right]\left[\frac{1}{\Lambda(k)}-\frac{1}{\Lambda^{\prime}(0) k}\right] d k
\end{aligned}
$$

Заметим, что функция $\Lambda(k)$ нечетная, поэтому распределение электрического поля в полупространстве можно представить в виде

$$
\frac{e(x)}{e(0)}=\frac{1}{\Lambda^{\prime}(0)}+\frac{2}{\pi} \int_{0}^{\infty} \sin (k x)\left[\frac{1}{\Lambda(k)}-\frac{1}{\Lambda^{\prime}(0) k}\right] d k .
$$



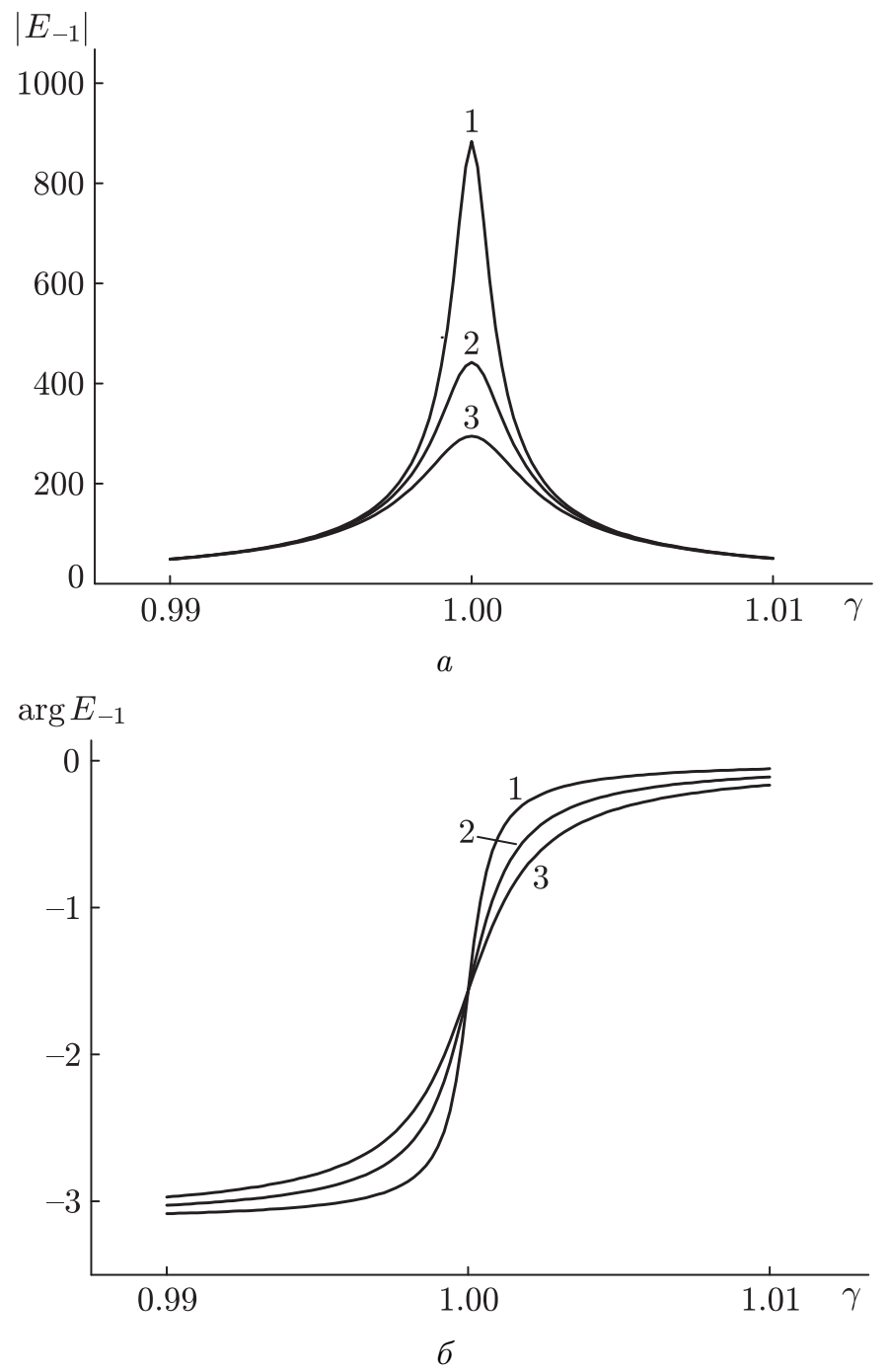

Рис. 1. Модуль (а) и аргумент (б) моды Друде вблизи плазменного резонанса. Кривые 1, 2, 3 отвечают соответственно значениям $\varepsilon=0.001,0.002,0.003$.

Отметим, что Ландау [2] выделяет постоянную Друде только у электрического поля.

Подчеркнем, что мода Друде отвечает нулю $k=0$ дисперсионной функции $\Lambda(k)$. Нетрудно видеть, что дисперсионная функция может иметь еще два конечных комплексных нуля $\pm k_{0}$ при соответствующих значениях параметра $\omega_{1}$. Покажем, например, что в низкочастотном пределе $(\omega \rightarrow 0)$ дисперсионная функция имеет два чисто мнимых нуля. В самом деле, при $\omega \approx 0$ на основании $(2.10),(2.11)$ имеем

$$
L_{1}(k)=\frac{2}{k} \operatorname{arctg} k, \quad L_{2}(k)=M_{1}(k)=0, \quad M_{2}(k)=\frac{2-L_{1}(k)}{k} .
$$

5 Теоретическая и математическая физика, т. 153, № 3, 2007 г. 

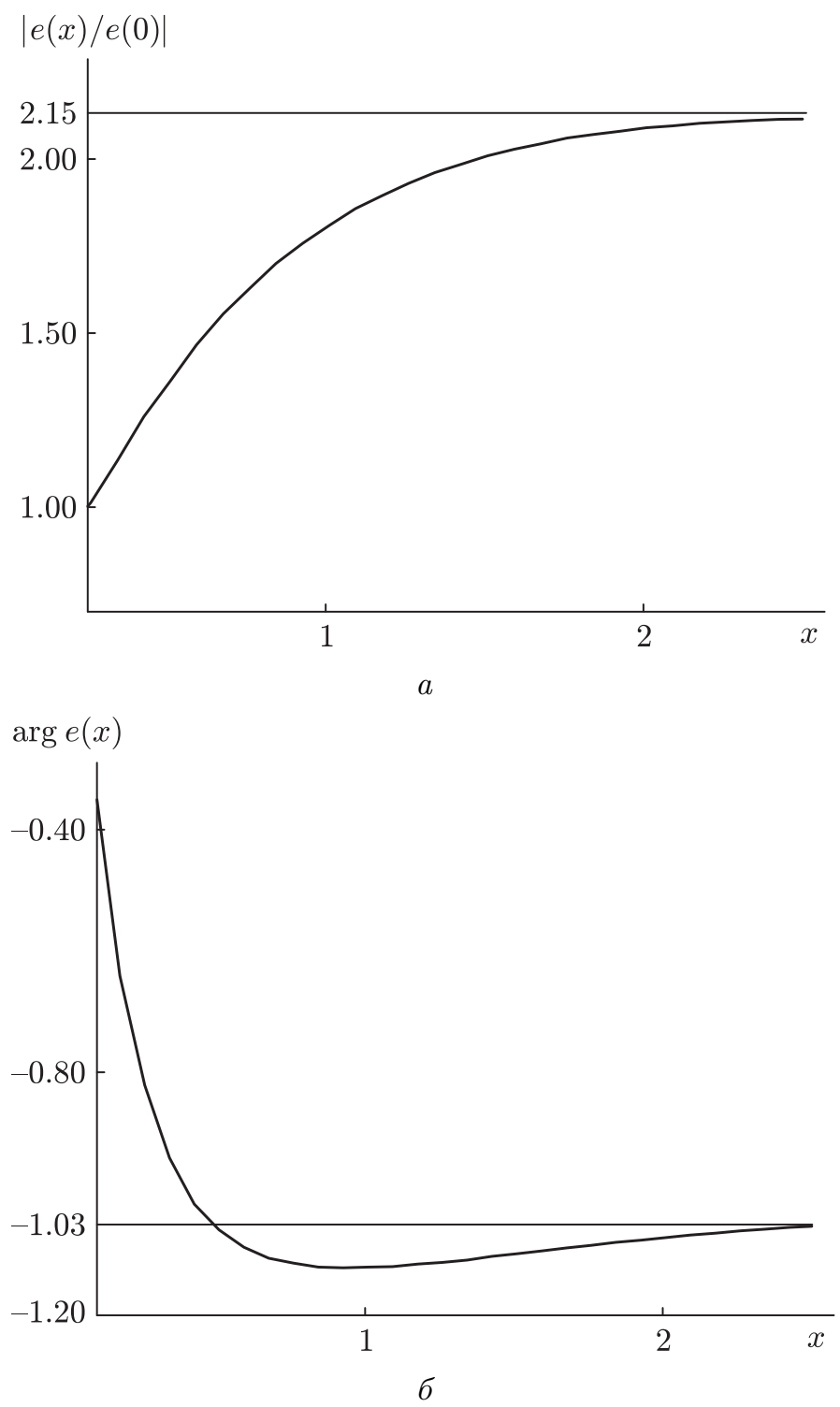

Рис. 2. Модуль (а) и аргумент (б) электрического поля.

Используя эти равенства, с учетом (2.12) получаем

$$
\begin{aligned}
T_{00}(k) & =\frac{\sqrt{\pi}}{4} L_{1}(k), & T_{01}(k) & =\frac{1}{2} L_{1}(k), \\
T_{10}(k) & =-i \frac{\sqrt{\pi}}{4} \frac{2-L_{1}(k)}{k}, & T_{11}(k) & =-\frac{i}{2} \frac{2-L_{1}(k)}{k} .
\end{aligned}
$$




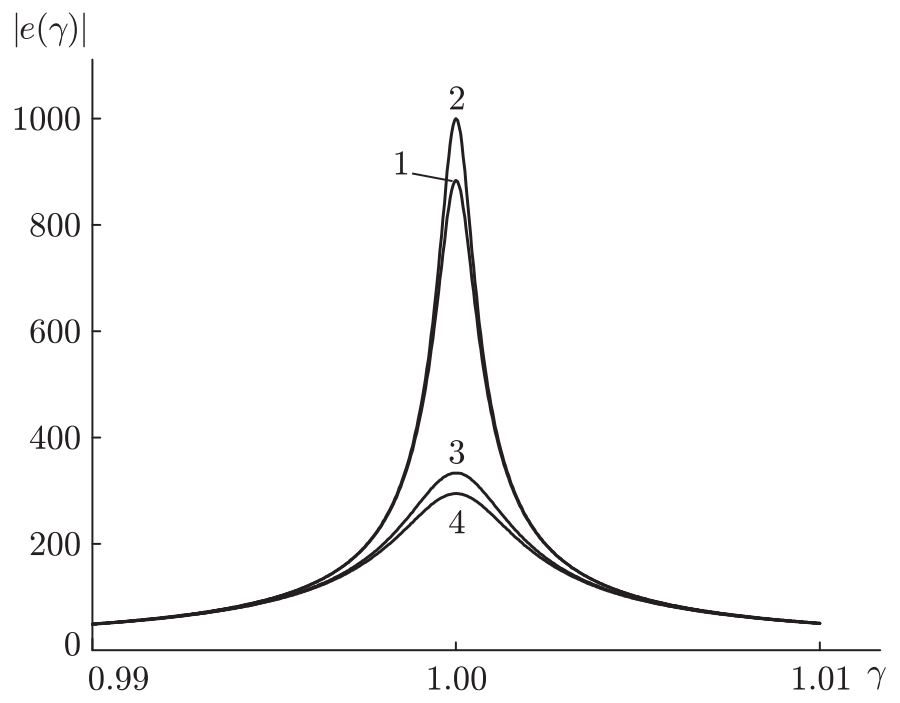

Рис. 3. Модуль моды Друде в случае частоты, пропорциональной скорости электронов (кривые 1,3 ), и в случае постоянной частоты столкновений (кривые 2,4$)$. Кривые 1,2 отвечают случаю $\varepsilon=0.001$, а кривые 3,4 - случаю $\varepsilon=0.003$.

Обозначим

$$
f_{0}(k)=\frac{\operatorname{arctg} k}{k}, \quad f(k)=1-f_{0}(k)
$$

Тогда

$$
1-T_{01}(k)=1-f_{0}(k)=f(k), \quad T_{10}(k)=-i \frac{\sqrt{\pi}}{2} \frac{f(k)}{k}, \quad T_{11}(k)=-i \frac{f(k)}{k} .
$$

Следовательно, выражение для дисперсионной функции в низкочастотном пределе принимает вид

$$
\Lambda(k)=k+\varkappa^{2} \frac{\sqrt{\pi}}{2 k}\left[f(k)+f_{0}(k)\right]=k+\frac{\sqrt{\pi} \varkappa^{2}}{2 k},
$$

или

$$
\Lambda(k)=k+\frac{2 \omega_{1 \mathrm{p}}^{2}}{k}=k+\frac{9 \pi \omega_{\mathrm{p}}^{2}}{8 \nu^{2} k}=k+\frac{9 \pi}{8 k \varepsilon^{2}} .
$$

Значит, дисперсионное уравнение имеет два чисто мнимых нуля $\pm k_{0}$, причем $k_{0}=$ $3 i \sqrt{\pi} /(2 \sqrt{2} \varepsilon), \varepsilon=\nu / \omega_{\mathrm{p}}$.

В окрестности нуля $k_{0}$ для дисперсионной функции имеем $\Lambda(k)=\Lambda^{\prime}\left(k_{0}\right)\left(k-k_{0}\right)$, $k \rightarrow k_{0}$. Согласно лемме Жордана с учетом того, что в рассматриваемой задаче $x>0$, получаем

$$
\frac{1}{2 \pi i} \int_{-\infty}^{\infty} \frac{e^{i k x}}{k-k_{0}} d k=e^{i k_{0} x}, \quad \operatorname{Im} k_{0}>0
$$


Таким образом, для распределения электрического поля в полупространстве (3.9) получено следующее выражение:

$$
\begin{aligned}
\frac{e(x)}{e(0)}= & \frac{1}{\Lambda^{\prime}(0)}+\frac{2 e^{i k_{0} x}}{\Lambda^{\prime}\left(k_{0}\right)}+ \\
& \quad+\frac{2}{\pi} \int_{0}^{\infty} \sin (k x)\left[\frac{1}{\Lambda(k)}-\frac{1}{\Lambda^{\prime}(0) k}-\frac{1}{\Lambda^{\prime}\left(k_{0}\right)\left(k-k_{0}\right)}\right] d k .
\end{aligned}
$$

Нулю $k_{0}$ отвечает следующее решение исходной системы $(1.15),(1.16)$ :

$$
e_{k_{0}}(x)=\frac{2 e(0) e^{i k_{0} x}}{\Lambda^{\prime}\left(k_{0}\right)}, \quad h_{k_{0}}(x, \mu, C)=e^{i k_{0} x} \Phi\left(k_{0}, \mu, C\right),
$$

где

$$
\Phi\left(k_{0}, \mu, C\right)=\frac{2 e(0)}{\Lambda^{\prime}\left(k_{0}\right)\left(1+i k_{0} \mu-i \omega_{1} / C\right)}\left[\mu+\frac{T_{11}\left(k_{0}\right)}{1-T_{01}\left(k_{0}\right)}\right] .
$$

Решение, отвечающее нулю $k_{0}$, естественно назвать решением Дебая. Это решение описывает экранированное электрическое поле в плазме.

Функцию распределения электронов с помощью моды Дебая аналогично (3.10) можно представить в следующем виде:

$$
\begin{aligned}
& \frac{h(x, \mu, C)}{e(0)}=\Phi_{0}(\mu, C)+e^{i k_{0} x} \Phi\left(k_{0}, \mu, C\right)+ \\
& \quad+\frac{1}{\pi i} \int_{-\infty}^{\infty} e^{i k x}\left[\Phi(k, \mu, C)-\frac{\Phi_{0}(\mu, C)}{k}-\frac{\Phi\left(k_{0}, \mu, C\right)}{k-k_{0}}\right] d k
\end{aligned}
$$

где

$$
\Phi_{0}(\mu, C)=\Phi(0, \mu, C)=\frac{\mu C}{\Lambda^{\prime}(0)\left(C-i \omega_{1}\right)} .
$$

\section{4. ЗАКЛЮЧЕНИЕ}

Впервые поставлена и аналитически решена задача о поведении в полупространстве столкновительной плазмы с частотой столкновений, пропорциональной модулю скорости электронов. Выявлена структура экранированного электрического поля в плазме и функции распределения электронов.

На рис. 1 представлены графики зависимости модуля и аргумента относительной моды Друде $E_{-1}=E_{-1}(\gamma, \varepsilon), E_{-1}(\gamma, \varepsilon)=1 / \Lambda^{\prime}(0, \gamma, \varepsilon)$ вблизи плазменного резонанса $\left(\omega \approx \omega_{\mathrm{p}}\right)$ от величины $\gamma=\omega / \omega_{\mathrm{p}}$. Модуль моды резко возрастает при $\omega=\omega_{\mathrm{p}}$, его значение тем больше, чем меньше значение параметра $\varepsilon=\nu / \omega_{\mathrm{p}}$. Аргумент моды при этом меняется от $-\pi$ до 0.

На рис. 2 представлены графики профилей модуля электрического поля и его аргумента в полупространстве $x>0$ при $\gamma=1, \varepsilon=0.5$. При возрастании координаты $x$ графики асимптотически "выходят" на значения, равные соответственно модулю и аргументу постоянной Друде.

Для сравнения поведения плазмы в случаях постоянной и переменой частоты столкновений приведен рис. 3, на котором изображен модуль моды Друде в случае 
частоты, пропорциональной скорости электронов $e_{v}(\gamma, \varepsilon)=E_{-1}(\gamma, \varepsilon)$ (кривые 1,3$)$, и в случае постоянной частоты столкновений (см. [4], [5])

$$
e_{c}(\gamma, \varepsilon)=\frac{\gamma(\gamma+i \varepsilon)}{2(\gamma-1)+i \varepsilon+(\gamma-1)(\gamma-1+i \varepsilon)}
$$

(кривые 2, 4).

Для количественного описания различия рассматриваемых случаев введем функцию относительного отклонения

$$
O(\gamma, \varepsilon)=\frac{e_{c}(\gamma, \varepsilon)-e_{v}(\gamma, \varepsilon)}{e_{v}(\gamma, \varepsilon)} \cdot 100 \%
$$

Наибольшее различие случаев постоянной и переменной частоты столкновений наблюдается на плазменном резонансе, т.е. при $\omega=\omega_{\mathrm{p}}(\gamma=1)$. В этой ситуации отклонение мало зависит от $\varepsilon$ (при малых $\varepsilon$ ). Так, $O(1,0.001)=O(1,0.003)=13.2 \%$. Однако уже при $\gamma=1.001$, т.е. при $\omega=1.001 \omega_{\mathrm{p}}$, имеем $O(1.001,001)=2.8 \%$, а $O(1.001,0.003)=9.3 \%$. При дальнейшем увеличении $\gamma$ наблюдается уменьшение функции отклонения. Так, $O(1.01,0.001)=0.036 \%, O(1.01,0.003)=0.317 \%$.

\section{Список литературы}

[1] А. А. Власов, ЖКЭТФ, 8:3 (1938), 291.

[2] Л. Д. Ландау, "О колебаниях электронной плазмы”, Собрание трудов, т. 2, Наука, М., 1969, 7; ЖЭТФ, 16 (1946), 547.

[3] В. М. Гохфельд, М.А. Гулянский, М.И. Каганов, А. Г. Плявенек, ЖЭТФ, 89:3(9) (1985), 985.

[4] А. В. Латышев, А. А. Юшканов, Изв. РАН. Сер. МЖГ, 2006, № 1, 165.

[5] А. В. Латышев, А. А. Юшканов, Ж ВМиМФ, 47:1 (2007), 121.

[6] А. В. Латышев, А. А. Юшканов, ТМФ, 147:3 (2006), 487.

[7] А. В. Латышев, А. А. Юшканов, ФTT, 48:12 (2006), 2113.

[8] А. В. Латышев, А. А. Юшканов, Физика плазмы, 32:11 (2006), 1021.

[9] В. П. Силин, А. А. Рухадзе, Электромагнитные свойства плазмы и плазмоподобных сред, Госатомиздат, М., 1961.

[10] В. В. Веденяпин, Кинетические уравнения Больимана и Власова, Физматлит, М., 2001.

[11] Е. М. Лифшиц, Л. П. Питаевский, Физическая кинетика, Наука, М., 1979.

Поступила в редакцию 28.12.2006, после доработки 26.03.2007 